\title{
ABSOLUTELY PURE MODULES
}

\section{B. H. MADDOX}

Introduction. In this paper several properties of absolutely pure modules are given. It is shown that absolutely pure and injective are equivalent properties for modules over Dedekind rings. However, it is proved that absolutely pure and injective are not equivalent properties for modules over rings which are not Noetherian. That every module has a maximal absolutely pure submodule is also established. A sufficient condition for the uniqueness of a maximal absolutely pure submodule is also given.

This paper constitutes a portion of the author's doctoral dissertation written at the University of South Carolina where he held a Cooperative Graduate Fellowship. The writer is indebted to Professor Edgar Enochs who suggested this topic and directed its development while providing sufficient inspiration and assistance and, most of all, exhibiting infinite patience.

In this paper all rings will have a unit and all modules will be unitary. $A$ will always denote a ring. We agree that if $E^{\prime}$ is a submodule of $E$ and $v: E^{\prime} \rightarrow E$ is the canonical injection then the map $1 \otimes v: F \otimes E^{\prime}$ $\rightarrow F \otimes E$ will be called the canonical map where $1: F \rightarrow F$ is the identity map of $F$. If the canonical map is an injection for all $F$, then $E^{\prime}$ is said to be a pure submodule of $E$. Observe that if $E^{\prime}$ is a pure submodule of $E$ then $a E \cap E^{\prime}=a E^{\prime}$ for all nonzero $a \in A$ by examining the diagram

$$
0 \rightarrow A / A a \otimes E^{\prime} \rightarrow A / A a \otimes E \rightarrow A / A a \otimes E / E^{\prime} \rightarrow 0
$$

where all of the maps are canonical. Immediately, a pure submodule of a divisible module (over an in tegral domain) is divisible. Hence this definition of purity generalizes the older notion. It can be shown the two properties are equivalent for modules over principal ideal domains.

An $A$-module $E$ is said to be absolutely pure if $E$ is a pure submodule of every module which contains $E$ as a submodule. For brevity, " $F$ contains $E$ " will always mean " $F$ contains $E$ as a submodule."

The first result is a generalization of Lemma 2 in $[4$, p. 15].

Lemмa 1. Suppose $E, F$ and $G$ are left $A$-modules such that $E \subset F \subset G$. If $E$ is pure in $G$ and $F / E$ is pure in $G / E$ then $F$ is pure in $G$.

Received by the editors September 27, 1965 and, in revised form, August 4, 1966. 
Proof. Let $H$ be any right $A$-module and chase the following commutative diagram (where all of the maps are the obvious ones).

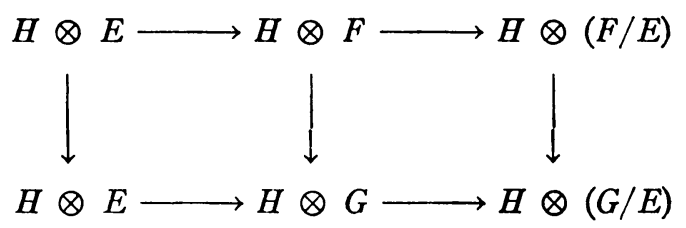

Using Lemma 1 we conclude that if $E^{\prime}$ and $E^{\prime \prime}$ are absolutely pure left $A$-modules and $0 \rightarrow E^{\prime} \rightarrow E \rightarrow E^{\prime \prime} \rightarrow 0$ is an extension of $E^{\prime}$ by $E^{\prime \prime}$ then $E$ is an absolutely pure left $A$-module. Therefore the direct sum of a finite number of absolutely pure left $A$-modules is an absolutely pure left $A$-module.

Lemma 2. If $E^{\prime}$ is a direct summand of a left $A$-module $E$ then $E^{\prime}$ is a pure submodule of $E$.

Proof. Immediate.

Lemma 3. If $E$ is an injective left $A$-module then $E$ is an absolutely pure left $A$-module.

Proof. Apply Lemma 2.

An immediate result of Lemma 3 and the observation following the definition of a pure submodule is that a torsion-free module $E$ over an integral domain $A$ is divisible if and only if $E$ is absolutely pure.

The following theorem can now be established.

Theorem 1. If $E$ is a module over a Dedekind domain $A$ then the following statements are equivalent:

(a) E is absolutely pure.

(b) $E$ is injective.

(c) $E$ is divisible.

Proof. Follows from [3, p. 134].

Lemma 4. If $E^{\prime}$ is a pure submodule of an absolutely pure left $A$ module $E$ then $E^{\prime}$ is an absolutely pure left $A$-module.

Proof. Let $F$ be any left $A$-module containing $E^{\prime}$. Form the direct sum, $F \oplus E$, of $F$ and $E$ and let $D$ denote the submodule of $F \oplus E$ generated by all elements of the form $(x,-x)$ where $x$ is in $E^{\prime}$. Write $(F \oplus E) / D=G$. Then by suitable identifications $E^{\prime} \subset F \subset G$ and $E^{\prime} \subset E \subset G$. Now $E^{\prime}$ is a pure submodule of $G$ for $E^{\prime}$ is pure in $E$ and $E$ is pure in $G$. Thus for any right $A$-module $H$ the canonical map $H \otimes E^{\prime} \rightarrow H \otimes G$ is an injection. However $H \otimes E^{\prime} \rightarrow H \otimes G$ is the com- 
position of the canonical maps $H \otimes E^{\prime} \rightarrow H \otimes F$ and $H \otimes F \rightarrow H \otimes G$. Hence $H \otimes E^{\prime} \rightarrow H \otimes F$ is an injection so $E^{\prime}$ is pure in $F$.

So, by virtue of Lemmas 3 and 4 , to prove a module is absolutely pure it suffices to prove it is pure in its injective envelope. Also, any direct summand of an absolutely pure module is absolutely pure.

THEOREM 2. If $\left(E_{\alpha}, f_{\beta \alpha}\right)$ is an inductive system of absolutely pure left $A$-modules and every $f_{\beta \alpha}$ is an injection then the inductive limit, $\lim E_{\alpha}$ is an absolutely pure left $A$-module.

Proof. The canonical maps $f_{\beta}: E_{\beta} \rightarrow \lim E_{\alpha}$ are injections. If $F$ is a left $A$-module containing $\rightarrow \lim E_{\alpha}$ and $\phi: \lim E_{\alpha} \rightarrow F$ is a map such that $\phi$ agrees with $\phi_{\beta}$ on $E_{\beta}$ where $\phi_{\beta}: E_{\beta} \rightarrow F$ is an injection for every $\beta$ then $\phi$ is an injection. Now, let $H$ be any right $A$-module. It is known [2, Corollary 3 to Proposition 12, p. 146] that $H \otimes \rightarrow \lim E_{\alpha}$ is isomorphic to $\lim \left(H \otimes E_{\alpha}\right)$ and using this isomorphism we have the commutative diagram $\otimes$

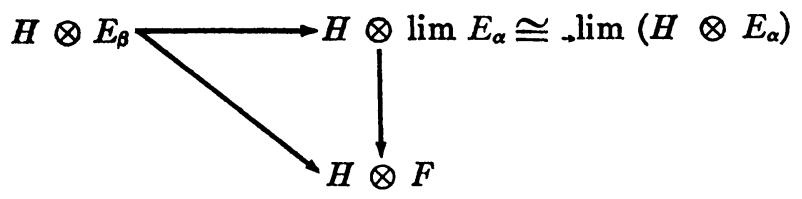

where all of the maps are canonical. So by the second statement $1_{H} \otimes \phi$ is an injection because $1_{H} \otimes \phi_{\beta}$ is an injection for every $\beta$.

Corollary. Let $\left(E_{i}\right)_{i \in I}$ be a family of left $A$-modules and $\bigoplus_{i \in I} E_{i}$ be their direct sum. Then $\bigoplus_{i \in I} E_{i}$ is absolutely pure if and only if $E_{i}$ is absolutely pure for each $i$ in $I$.

Thus we see that although injective and absolutely pure are equivalent properties for modules over Dedekind rings they are not equivalent over rings which are not Noetherian. This follows for $H$. Bass [1] has shown that Noetherian rings are characterized by the direct sum of an infinite family of modules being injective if and only if each module of the family is injective.

ExAmple. Let $F_{i}$ denote a field for each $i$ in $I$ where $I$ is an infinite set. Let $B=\bigoplus_{i \in I} F_{i}$ and $A=\prod_{i \in I} F_{i}$. Consider $B$ and $A$ as $A$ modules. $B$ is absolutely pure but $B$ is not injective.

Example. Let $A_{i}=Z / 4 Z$ for $i \in N$ and $A=\prod_{i \in N} A_{i}$. Let $B$ be those elements of $A$ of finite support, i.e., $B=\bigoplus_{i \in N} A_{i}$. Let $C$ be those elements $a \in A$ such that $\operatorname{pr}_{i}(a)=0$ or $\operatorname{pr}_{i}(a)=2$ for almost all $i \in N$ where $\operatorname{pr}_{i}: A \rightarrow A_{i}$ is the canonical projection. Clearly $A, B$ and $C$ can be considered as $A$-modules in a natural fashion.

It can be shown that $B$ is an absolutely pure $A$-module and that 
$B$ is essential in $A$ hence essential in $C$ for $B \subset C \subset A$. However it can also be shown that $C$ is not absolutely pure. Thus we have an example of an absolutely pure module $B$ which is contained essentially in a module $C$ where $C$ is not an absolutely pure module.

Also $B$ is not injective for $B$ is contained essentially in a module $C$ where $C \neq B$. So we also have an example of an absolutely pure module which is not injective.

It also turns out that $B$ is flat so a module being flat and absolutely pure does not always imply that the module is injective.

Given a left $A$-module $E$, consider the set (S) of all submodules of $E$ that are absolutely pure modules. Observe that (f) is not empty, for

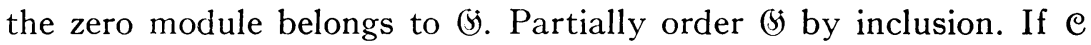
is a chain in (5) then $\bar{E}=\bigcup_{E^{\prime} \in \mathbb{C}} E^{\prime}$ is an absolutely pure left $A$-module by Theorem 2 .

Now by applying Zorn's Lemma we know that every module has a maximal absolutely pure submodule. Of course this result presents the question of whether such a maximal submodule is unique. It follows easily that if $A$ is a ring such that every quotient module of an absolutely pure left $A$-module is absolutely pure then each left $A$-module has a unique maximal absolutely pure submodule.

\section{REFERENCES}

1. H. Bass, Global dimension of rings, Ph.D. Thesis, Univ. of Chicago, Chicago, Ill., 1959.

2. N. Bourbaki, Algebre, Chapter 2, Algebre Lineaire, Hermann, Paris, 1962.

3. H. Cartan and S. Eilenberg, Homological algebra, Princeton Univ. Press, Princeton, N. J., 1956.

4. I. Kaplansky, Infinite Abelian groups, Univ. of Michigan Press, Ann Arbor, Mich., 1954.

Florida Presbyterian College 